\title{
NOTE ON THE APPLICATION OF COMPOUND POISSON PROCESSES TO SICKNESS AND ACCIDENT STATISTICS
}

\section{Carl Philipson}

Sweden

1. In order to fix our ideas an illustration of the theory for (a) a general elementary random process, (b) a compound Poisson process and (c) a Polya process shall be given here below following Ove Lundberg (On Random Processes and Their Application to Accident and Sickness Statistics, Inaug. Diss., Uppsala I940).

(a) The general elementary random process.

Let the continuous parameter $t^{*}$ be measured on an absolute scale from a given point of zero and consider the random function $N^{*}\left(t^{*}\right)$ which takes only non-negative and integer values with $N^{*}(0)=0$. This function constitutes a general elementary random process for which the conditional probability that $N^{*}\left(t^{*}\right)=n$ relative to the hypothesis that $N^{*}\left(t_{0}^{*}\right)=m$ shall be denoted $P_{m n}^{*}\left(t_{0}^{*}, t_{1}^{*}\right)$, while the absolute probability that $N^{*}(t)=n$ i.e. $P_{o n}^{*}\left(\mathrm{o}, t^{*}\right)$ shall be written $P_{n}^{*}\left(t^{*}\right)$. If quantities of lower order than $d t^{*}$ are neglected, we may write $p_{n}^{*}\left(t^{*}\right) d t^{*}$ for the conditional probability that $N^{*}\left(t^{*}+d t^{*}\right)=n+$ I relative to thehyp othesis that $N^{*}\left(t^{*}\right)=n$, i.e. $P_{n, n+1}^{*}\left(t^{*}, t^{*}+d t^{*}\right) . p_{n}^{*}\left(t^{*}\right)$ is the intensity function of the process which is assumed to be a continuous function of $t^{*}$ (the condition of existence for the integral over the given interval of $t^{*}$ for every $n>m$ may be substituted for the condition of continuity). The expectations for an arbitrary but fix value of $t^{*}$ of $N^{*}\left(t^{*}\right)$ and $p^{*}\left(t^{*}\right)$ will be denoted by the corresponding symbol with a bar so that

$$
\bar{N}^{*}\left(t^{*}\right)=\sum_{n=0}^{\infty} n P_{n}^{*}\left(t^{*}\right) \text { and } \bar{p}_{n}^{*}(t)=\sum_{n=0}^{\infty} p_{n}^{*}\left(t^{*}\right) P_{n}^{*}\left(t^{*}\right) .
$$

If $p_{n}^{*}\left(t^{*}\right) / n$ is uniformly bounded for all $n$ in the interval $0 \leq t^{*}<T^{*}$, where $T^{*}$ is an arbitrary but fix value of $t^{*}$, we have i.a. that 


$$
\bar{N}^{*}\left(t^{*}\right)=\int_{0}^{t^{*}} \bar{p}^{*}(u) d u
$$

By the transformation $t=\bar{N}^{*}\left(t^{*}\right)$ in the functions $N^{*}\left(t^{*}\right)$ $P_{n}^{*}\left(t^{*}\right), p_{n}^{*}\left(t^{*}\right)$ and $\bar{p}_{n}^{*}\left(t^{*}\right)$ functions will be obtained which will be designated $N(t), P_{n}(t), p_{n}(t)$ and $\bar{p}_{n}(t)$ respectively. By putting $t_{0}=N^{*}\left(t_{0}^{*}\right), t_{1}=N^{*}\left(t_{1}^{*}\right)$ the transformation in $P_{m n}^{*}\left(t_{0}^{*}, t_{1}^{*}\right)$ leads to a corresponding function which will be denoted $P_{m n}\left(t_{0}, t_{1}\right) \cdot t$ is said to be measured in the operational scale. By ( $\mathrm{x}$ a) the transformation to the operational scale can be defined by

$$
t=\int_{0}^{t^{*}} \overline{p_{n}^{*}}\left(u^{*}\right) d u^{*}
$$

(b) The compound Poisson process.

These processes form a particular class of the elementary random processes defined in (a) for which the absolute probabilities fulfil the following relation with the parameter measured on the operational scale:

$$
P_{n}(t)=\int_{0}^{\infty} \frac{(t x)^{n}}{n !} \mathrm{e}^{-t x} d U(x)=\frac{(-t)^{n}}{n !} P_{0}^{(n)}(t),
$$

where the integral is a Laplace-Stieltje's integral, $P_{0}^{(n)}(t)$ denotes the $n^{\text {th }}$ derivative of $P_{0}(t)$ with respect to $t$, and $U(x)$ has the properties of a distribution function defining the unconditioned risk distribution. If, on the other hand, $P_{\mathbf{0}}(t)$ is a given function which is completely monotonic, i.e. $(-t)^{n} P^{(n)}(t) \geq 0$, the first part of the equality follows from a general theorem and $U(x)$ is, then, a nondecreasing function and bounded in every finite interval. (Bernstein, Acta Math., 52, pp. I-66; Feller, Duke Math. Journ. I959, pp. 66I-674). If, in addition, $\lim _{t \rightarrow 0} P_{0}(t)=\mathrm{I}, P_{n}(t)$ defines a compound Poisson process in the generalized sense (i.e. points where $P_{n}(t)=0$ are disregarded).

The intensity function, $p_{n}(t)$ of a compound Poisson process has i.a. the following properties:

$$
p_{m}\left(t_{0}\right)=\sum_{n=m}^{\infty} p_{n}\left(t_{1}\right) P_{m n}\left(t_{0}, t_{1}\right), m<n, t_{0}<t_{1}
$$


For $m=0(2$ a) leads, after insertion in ( $\mathrm{I}$ b), to

$$
t=\int_{0}^{t *} p_{0}\left(u^{*}\right) d u^{*}
$$

for the transformation to the operational scale.

$$
p_{n}(t)=-P_{0}^{(n+1)}(t) / P_{0}^{(n)}(t),
$$

where $P_{0}^{(n)}(t)$ is defined under (2).

$$
\begin{gathered}
p_{m}\left(t_{0}\right)=\frac{\mathrm{I}}{t_{1}-t_{0}} \sum_{n=m}^{\infty}(n-m) P_{m n}\left(t_{0}, t_{1}\right), m=0, \mathrm{I} \ldots \\
p_{n+1}(t)=p_{n}(t)-\frac{p_{n}^{\prime}(t)}{p_{n}(t)}, n=0, \mathrm{I} \ldots
\end{gathered}
$$

by which, if $p_{0}(t)$ is given by the relation $-P_{0}^{\prime}(t)=p_{0}(t) P_{0}(t)$, the functions $p_{n}(t)$ are uniquely defined for all $n$ according to $(2 \mathrm{c})$. We have, further, the following properties of the probability functions

$$
P_{m n}\left(t_{0}, t_{1}\right)=\left(\begin{array}{c}
n \\
m
\end{array}\right)\left(\frac{t_{0}}{t_{1}}\right)^{m}\left(\mathrm{I}-\frac{t_{0}}{t_{1}}\right)^{n-m} \cdot \frac{P_{n}\left(t_{1}\right)}{P_{m}\left(t_{0}\right)}, m<n, t_{0}<t_{1},
$$

where the binomial factor represents the conditional probability that $m$ changes have occurred in the interval $\left(0, t_{0}\right)$ of the parameter relative to the hypothesis that $n$ changes have occurred in the interval $\left(0, t_{1}\right)$, where $t_{1}>t_{0}$ (inverse probability).

$$
t_{k} \beta_{k}=\sum_{k}^{\infty} n(n-\mathrm{I}) \ldots(n-k+\mathrm{I}) P_{n}(t)
$$

where $\beta_{k}$ are the moments about zero of $U(x)$.

[Lundberg l.c. (77), (78), (97), (8I), (88), (94), (I25)]

(c) The Polya process

A compound Poisson process defined by (2) will be called a Polya process, if $U(x)$ is defined by the Pearson density function Type III (Lundberg, 1.c. pp. I8-19). It can be derived from a Polya-Eggen- 
berger urn scheme by ascribing a time interval, $t / h$, to each drawing and by making a limit passage for $h$ tending to infinity under two auxiliary conditions (1.c. pp. I6-I7). The same process is obtained from Lexis' urn scheme either by assuming the risks to form a continuous set distributed with Pearson density function Type I, which leads to the Polya-Eggenberger's probabilities, and a following limit passage (1.c. pp. I3-I4) or by the direct application of a limit passage according to Poisson to the Lexis' probabilities (Ammeter, Skand. Akt. Tidskr. I948, pp. I75, I78). Ammeter deducts, also (l.c. (I2)) a process which may be considered a transform of several Polya processes with equal variance of $U(x)$. This transform gives, at the end of each finite unit period, the same result as a process constructed by Arfwedson (Skand. Akt. Tidskr., 1955, pp. 97-100) based on the assumption that the probability of more than one claim in the interval $(t, t+d t)$ of the parameter is of the order $d t$ (cf. Philipson, Skand. Akt. Tidskr., I956, pp. 26-37; Trans. XVth Int. Congr. Act., II, New York I957, pp. 264-279).

The intensity function, $p_{n}(t)$, of a Polya process is defined by the following relation:

$$
p_{n}(t)=(\mathbf{I}+b n) /(\mathbf{I}+b t),
$$

where $b$ is the variance of $U(x)$ (Lundberg, l.c., (I5)). Consequently by (2) the regression of $N\left(t_{1}\right)-N\left(t_{0}\right)$ on $N\left(t_{0}\right)$ is linear for a Polya process. The correlation coefficient between these values of a general compound Poisson process with mean and variance of $U(x)$ equal to $I, b$ respectively depends only on $b, t_{0}, t_{1}$. Therefore, the first approximation to every compound Poisson process-the "best" approximation according to the principle of least square-has the form of a Polya process with $U(x)$ of the same mean and variance (Lundberg, l.c., p. III).

2. Thyrion (The ASTIN Bull., I part III, pp. I42-I62; Trans. $\mathrm{XVI}^{\text {th }}$ Int. Congr. Act., II, Brussels I960, pp. 25-36) reports on investigations of the distribution of claims in Motor Insurance. In bcth of the papers formulae are deducted, which either are identical, with those quoted above or are direct consequences of them, e.g. the last formula on page 28 of Thyrion's paper to the Congress of Brussels is by the last membrum of (2) and (2 c) a direct consequence of $(2 \mathrm{~d})$. On page 26 of the same paper he takes 
into account the heterogeneity between groups, assuming that the conditional probability of one claim in the interval $\left(t^{*}, t^{*}+d t^{*}\right)$ of the parameter relative to the hypothesis that $n$ claims have occurred in the interval $\left(0, t^{*}\right)$ can be expressed $\lambda \mu\left(t^{*}\right)$ where $\lambda$ is allowed to vary from group to group and $\mu\left(t^{*}\right)$ is identically the same for all groups for an arbitrary but fix value of $t^{*}$. The development on this point seems to have been made more rigorous by taking the relations ( $\mathrm{I} b),(2 \mathrm{~b})$ into consideration.

On pages $I 5^{I-I} 5^{2}$ of the Astin paper and on page 29 of the paper to the Congress Thyrion refers to Hofmann (Bull. des actuaires suisses, 55, pp. 499-576) by quoting the last relation in (2), which holds for every compound Poisson process and by giving a particular expression for $P_{\mathbf{0}}(t)$, which defines a subclass of these processes. In the subsequent section the theory expounded by Hofmann will be separately considered.

3. Hofmann gives, first, a generalization of an one-dimensional compound Poisson process to such a process constituted by two random functions $(M(\vartheta), N(\vartheta)$, which may be mutually dependent or independent. The probability that $M(s)=m, N(t)=n$ is here denoted $\pi(m, n, s, t)$ and can be defined by the relation

$$
\pi(m, n, s, t)=\int_{0}^{\infty} \int_{0}^{\infty} e^{-k s-\lambda t} \frac{(k s)^{m}}{m !} \cdot \frac{(\lambda t)^{n}}{n !} d U(k, \lambda)
$$

Hofmann deducts for an one-dimensional compound Poisson process a general expression for $P_{n}(t)$ in terms of $p_{0}(t)$ (in his notations designated $\left.q \tau^{\prime}(t)\right)$ and a recurrence formula for $P_{n}(t)$. He defines, then, a subclass of the class of compound Poisson processes by the approach:

$p_{0}(t)=q\left(\mathrm{I}+\frac{b t}{\alpha}\right)^{\alpha}$ where $q, b, \alpha$ are positive constants.

This relation is inserted into the expression for $P_{n}(t)$ and in the recurrence formula. The recurrence formula for the process defined in (5) (4.Io in Hofmann's paper) has been directly deducted by Thyrion (Astin Bull., p. I56; Trans. XVIth Int. Congr. Act., p. 30) by the method used by Hofmann for the general compound Poisson process. 
It is easily seen that (5) for $\alpha=0$ defines a Poisson process and for $\alpha=\mathrm{I}$ a Polya process. Observing that the characteristic function corresponding to $U(x)$ is defined by $P_{0}(-i z)=\exp .\left[-\int_{0}^{i z} p_{0}(v) d v\right]$ Hofmann deducts $d U(x)$ for different values of $\alpha$. For $\alpha=0,1, \infty$ $U(x)$ is defined by I for $x \geqslant q$, o for $x<q$; by the Pearson density function Type III and the Poisson probability function respectively. For $\alpha=2 d U(x)$ is equal to a modified Bessel function and for $0<\alpha<$ I equal to a product of an exponential function and a stable frequency function being a particular case of such functions defined by Lévy (Théorie de l'addition des variables aléatoires, Paris I937). For I $<\alpha<\infty d U(x)$ cannot generally be given in a closed form, but is given in the form of a power series. For the particular case $\alpha=1 / 2$, finally, $U(x)$ is defined by the frequency function:

$$
\frac{q i}{\sqrt{2 \pi b}} e^{q / b} x^{-3 / 2} \exp .\left[-\frac{\mathrm{I}}{2 b}\left(x+\frac{q^{2}}{x}\right)\right]
$$

i.e. a product of Pearson's density functions Type III and V multiplied by a constant. (Hofmann, 1.c. pp. 526-529).

Hofmann (l.c. p. 53I) deducts further from the characteristic function corresponding to $U(x)$ of the process defined by (5) the $k_{k}$ th semi-invariant, $x_{k}$, of $U\{x\}$ for $k=I, \ldots ; x_{k}$ may be expressed by the following relation

$$
x_{k}=\left\{\begin{array}{l}
q \text { for } k=\mathrm{I} \\
q b^{k-1} \prod_{\nu=0}^{k-2}\left(\mathrm{I}+\frac{\nu}{\alpha}\right) \text { for } k>\mathrm{I} .
\end{array}\right.
$$

The mean and variance of $U(x)$ are, thus, equal to $q, q b$ respectively and the mean and variance of $P_{n}(t)$ for an arbitrary but fix value of $t$ equal to $q t, q t(\mathrm{I}+b t)$ respectively independently of $\alpha$. According to Lundberg (1.c. (I24), (I4)) the last mentioned values are applicable to every compound Poisson process, if, $q, q b$ denotes the mean and variance of a general unconditioned risk distribution. Thyrion gives in both papers quoted above the deductions of $(5 \mathrm{~b})$ according to Hofmann.

It can easily be proved by inserting ( 5 b) into Pearson's criterion 
(Elderton, Frequency Curves and Correlation, London 1927, pp. $50,53)$ that if $b>0.125 q,(5$ a) can be approximated by a Pearson density function Type VI. In this connection it might be remarked that the author of this note has, in a tentative application of Lundberg's theory to the growth of plants considered a random process constituted by the logarithm of the crop and with two parameters, the marginal processes being defined as Polya processes, found that the distribution of the value (or quantity) of the crop may be defined by a frequency function which approximately equals the Pearson density function Type VI. (Philipson, Skand. Akt. Tidskr., I955, pp. 228-23I, 234-235, 242-245).

Hofmann also gives an integral equation for $d U(x)$ of a process belonging to the subclass defined by (5 a) (1.c. pp. 529-530). Further, he gives a generalization of the subclass such as to contain also non-elementary processes. This generalization is developed for an example involving $U(x)$, for the number of changes, equal to Pearson Type III, and an exponential distribution for the size of one change. The deductions lead to similar results as those given by Lundberg with a general distribution of the size of one change (l.c. chapter VI).

4. In the numerical investigations reported by Hofmann the agreement between the estimated inverse probability and the binomial expression in $(2 f)$ is tested by the $\chi^{2}$-method (binomial test). The test gave $\chi^{2}$ equal to I0.5, xi. 6 for the two groups investigated (accidents within and outside working time) respectively with I6, I2 degrees of freedom respectively, corresponding to probabilities for higher values of $\chi^{2}$ of $83.9,44.6 \%$ respectively. Further, the differences between the variance of $U(x)$ for two adjoining periods within each of these groups have been calculated by $(5 b)$ and compared with their random errors (computed by a method by Tschuprow). The differences are found to be less than twice respectively one time the random error for the two groups. The hypothesis that the material can be described by a general compound Poisson process could, therefore, be accepted. The test of the agreement between the estimated $P_{m n}\left(t_{0}, t_{1}\right)$ with the expression in the right membrum of $(2 f)$ gave the same result. The frequencies for I-year periods can, however, not be assumed to be constant, the variation being due neither to a trend nor to a 
periodicity. It could, in fact, be explained by the assumption of a further distribution according to an idea by Ammeter (Bull. des actuaires suisses, 49).

The binomial test and a study of the estimates of the means and variances of the inverse probability function as applied by Lundberg to Sickness Insurance, at least for a part of the material investigated, lead to a rejection of the hypothesis of a compound Poisson process. The same result was obtained by a test of the estimated variances of $U(x)$ for different periods, based on an assumption of normal sampling distribution of these estimates. An explanation of the systematic deviations in $U(x)$ corresponding to the latter of these periods is given. Further, it is shown that time homogeneous processes would give greater differences between observed and calculated frequencies.

5. A comparison between the empirically ascertained frequencies with those calculated according to (5 a) with $q, b$ and $\alpha$ as estimated from the data and with the estimates of $q, b$ for $\alpha=\mathrm{I}$ was made in Hofmann's paper. In the first comparison the estimates $0.76603,5.7232$ were used for $\alpha$ of the two groups respectively. When using the estimated values for $\alpha, \chi^{2}$ was found to equal 9.06, 4.89 with 5,3 degrees of freedom respectively and, using $\alpha=\mathrm{I}, \chi^{2}$ was $9.54,5.57$ with 6,4 degrees of freedom respectively. These values correspond to probabilities of higher values for $\chi^{2}$ of ro.I, I7.2 $\%$ for estimated $\alpha: s$, and $\mathbf{I} 4.3,23 . \mathbf{I} \%$ respectively for $\alpha=\mathbf{I}$. Consequently, no choice with respect to $\alpha$ can be made on these tests. The comparison between the empirical frequencies of both groups--within and outside working time-with Hofmann's twodimensional example with marginal Polya-distributions lead to $\chi^{2}=\mathrm{I} 7.3$ for $\mathrm{I} 2$ degrees of freedom corresponding to a probability for higher value of $\chi^{2}$ of $13.2 \%$. This test supports a statement that models based on $\alpha=\mathrm{I}$ can be used for the graduation.

Thyrion has in his paper in the Astin Bulletin calculated the conditional means on different assumptions. Taking his figures for an earlier period of $\mathrm{I}-3$ years and a later period of $\mathrm{I}$ year the author of this note has calculated the relative differences between the calculated means with $b=q=0.25$ for $\alpha=\mathrm{I}$ and $\alpha=\mathrm{I} / 2$ as related to the former values. These differences are given in the following table. 
Relative differences in means calculated with $\alpha=\mathrm{I}$ and $\mathrm{I} / 2$

\begin{tabular}{lrrrr}
\hline$m$ & $t_{1}-t_{0}=$ & \multicolumn{1}{c}{ I } & \multicolumn{1}{c}{3} \\
\hline 0 & -0.025 & -0.060 & -0.096 \\
$\mathrm{I}$ & 0.075 & 0.090 & 0.098 \\
2 & $-0.02 \mathrm{I}$ & 0.045 & 0.070 \\
3 & -0.128 & -0.034 & 0.018 \\
4 & -0.175 & -0.109 & -0.040 \\
\hline
\end{tabular}

These figures ought to be compared with the random errors in the empirical frequencies used for the graduation. If we disregard the random error in $m$, the random errors in $n$ can be calculated on the basis of the variances of $P_{m, n}\left(t_{0}, t_{1}\right)$. Using the results of Lundberg (l.c. p. 97) for the "sub-process" of a Polya process expressing this probability as an absolute probability, these variances can be computed by the expression

$$
q \cdot \frac{\mathrm{I}+b m}{\mathrm{I}+b t_{0}}\left(t_{1}-t_{0}\right)\left[\mathrm{I}+\frac{b}{\mathrm{I}+b t_{0}}\left(t_{1}-t_{0}\right)\right]
$$

obtained from $q t(\mathrm{I}+b t)$ by the substitution of $\frac{\mathrm{I}+b m}{\mathrm{I}+b t_{0}}\left(t_{1}-t_{0}\right)$, $\frac{b}{\mathrm{r}+b m}$ for $t, b$ respectively.

Applied to Thyrion's material in his paper to the Congress and for $b=q=0.25$ this gives the following relative standard errors in the frequencies, if the random errors in $m$ are disregarded.

RELATIVE STANDARD ERRORS IN $n$

\begin{tabular}{crrrr}
\hline$m$ & $t_{1}-t_{0}=$ & $\mathrm{I}$ & 2 & 3 \\
\hline $\mathrm{O}$ & 0.015 & $0.0 \mathrm{I} 4$ & $0.0 \mathrm{I} 6$ \\
$\mathrm{I}$ & 0.038 & 0.030 & 0.028 \\
$\geq 2$ & 0.092 & 0.050 & 0.045 \\
\hline
\end{tabular}

Taking regard also to the standard errors in $m$ the figures of the last table should be multiplied by a factor exceeding $\sqrt{ } 2$. Therefore, it must be concluded that the material used for the graduation does not permit of a choice between $\alpha=I$ and $\alpha=I / 2$. If both these 
hypotheses had been adequately tested with the $\chi^{2}$-method the test would probably have lead to an acceptance of both hypotheses. Thyrion says that his reason for the choice of $\alpha=\mathrm{r} / 2$ is that "les ajustements faits avaient montré que le paramètre $\alpha$ était le plus généralement compris entre $o$ et $\mathrm{I}$ et pouvait moyennement être pris égale à $\mathrm{I} / 2$; nous adopterons cette valeur par mesure de simplification", (in his paper to the Congress p. 29). He discusses the same topic from the point of view of a passage to the limit of $P_{0}(t)$ for $t$ tending to infinity, in his earlier paper (pp. I54-I55). It shall be observed that the estimates of $\alpha$ are computed by the solution of the transcendental equation given by Hofmann and quoted by Thyrion (The ASTIN Bull., p. I53). Thyrion thinks, however, that this relation is chosen rather arbitrarily and concludes, therefore, that the ordinary $\chi^{2}$-method might not be applicable (l.c. p. I54). It might also be referred to the statement given in section 3 that the mean and variance of $U(x)$ and $P_{n}(t)$ are independent of $\alpha$, while the higher semi-invariants depend on $\alpha$. Consequently, a change of $\alpha$ leads to a change in the semi-invariants of higher order than the second order. It is well-known that a considerable number of observed cases must be demanded if the random errors in the semiinvariants of the $3^{\text {rd }}$ and $4^{\text {th }}$ order shall be small enough to permit of conclusions involving differences in these semi-invariants.

6. Lundberg says in his book (l.c. p. I6r) that "What our analysis properly yields is not an answer to the question as to whether or not the hypothesis strictly reproduces reality but the degree of approximation with which the random process reproduces reality. This is sufficient for us to be able to judge whether the process may be considered usable as a basis for re-grading sickness insurance premiums".

In spite of the fact that the tests of Lundberg's material have lead to a rejection of the hypothesis, we may use a particular form of $U(x)$ to describe the heterogeneity of a certain period. Such a description can always be made even though a compound Poisson process is not applicable, i.e. the hypothesis of a constant risk distribution cannot be accepted. The description of the heterogeneity implies that an endeavour is made to characterize the real course of the events with a special form of a compound Poisson process. Lundberg suggests that $U(x)$ shall be represented by the 
Pearson density function Type III and gives two graphs each representing the regression lines for the number of claims of one subperiod with respect to that of the other, for each one of the two longer periods investigated (l.c. pp. I62-I63). These graphs affords a better knowledge of the applicability of the Polya process, which is the only compound Poisson process with linear regression. These graphs may be directly compared with the corresponding graphs in Hofmann's paper (1.c. pp. 560-561). Taking regard to the larger random errors for higher number of claims the graphs both in Lundberg's book and in Hofmann's paper give the impression that the reality is fairly well represented by Polya processes. Finally, Lundberg compares estimates of the variance of $U(x)$ as computed from the estimated variance and correlation coefficient of $P_{n}(t)$ on the assumption that the process is a general compound Poisson process and from the estimate of $P_{0}(t)$ assuming the process to be a Polya process and obtains approximately $0.94,0.80$ and 0.84 for the first period and $0.46,0.47$ and 0.52 for the second period. Therefore, he tests the agreement between the observed frequencies with those calculated on the basis of a Polya process for each period with $b$ equal to $0.94 \mathrm{I} 6,0.457 \mathrm{I}$ respectively as obtained from the estimated variances of $P_{n}(t)$. He obtains $\chi^{2}=$ I4.I, I2.I with I4, Io degrees of freedom corresponding to probabilities for higher values of $\chi^{2}$ of $30-50,20-30 \%$ respectively. Lundberg concludes that "The heterogeneity of the two records may, thus, be said to be well described by a Polya distribution." Eggenberger (Bull. des actuaires suisses, I924, pp. 3I-I44) and Newbold (Journ. Roy Stat. Soc., 90, pp. 487-547) have come to the same result for similar records.

Finally, Lundberg compared, also the observed frequencies with those calculated on the assumption of $U(x)$ being a step function of 2 steps only ("a double Poisson"). The result of this test gave $\chi^{2}=$ 53.4 for 9 degrees of freedom with a probability for higher values of $\chi^{2}$ equal to $\mathrm{I} \%$, wherefore the hypothesis of a "double Poisson" must be rejected. (Cf., however, Depoid, Bull. Trim. de l'Inst. Act. Franc., 227, pp. I2I-I23).

Consequently, Lundberg suggests that the re-grading of sickness insurance premiums shall be based on a Polya process.

Hofmann has (1.c. pp. 546-548) discussed the theoretical deduc- 
tions by Greenwood and Yule (Journ. Rey. Stat. Soc., 83) and those of Neyman and Bates (Univ. Cal. Publ. Stat., I). The first-mentioned of these deductions is i.a. based on the hypothesis that the different individuals in a statistical group from the beginning have different probabilities, while Neyman assumes that $p_{n}(t)$ is equal to $z(n) /(\mathrm{I}+c t)$, where $z(n)>0$ and $c \geq 0$. Observing that by $(2 \mathrm{e})$ here above, the Polya process can be deducted on both of these assumptions, Hofmann says (p. 549) that "Der Nachteil der negativen Binominalverteilung" $\left(P_{n}(t)\right.$ of a Polya process) "liegt darein, dass sie sowohl als Ansteckungsverteilung" (Neyman) "wie zusammengesetzte Poisson Verteilung" (Greenwood and Yule) "interpretiert werden kann". The author of this note finds this feature to be an advantage. The numerical results may be approximately and rationally explained in terms of a Polya process independently, whether to our knowledge one or both of the hypotheses-accident proneness and heterogeneity-is true. As Lundberg pointed out (see above in the beginning of this section) we are more interested in an approximative but adequate description of the material than to test whether a hypothesis strictly reproduces reality. Also Hofmann seems to be more interested in a model apt to be used as a basis for the computations in accident insurance rather than in a tentative theoretical interpretation of the complicated psychological phenomen involved (1.c. p. 555). Thyrion states that a test of the agreement between theoretical and empirical values seems not to be necessary as the graduation even if not optimal seems to lead to a satisfactory description of the observed values (The ASTIN Bull. p. I54). On this point it might be of interest to refer to a discussion in Lundberg's book (1.c. pp. I30-I3I) according to which the accident proneness is likely to be of very little importance in comparison with the effect of heterogeneity. Hofmann has in the discussion of the graduation based on his example of a twodimensional Polya process presented an interesting statistical interpretation of the partition of the risk in his two groups into two components, the first component of each group constitutes a probability which is independent of the other group, while the second component of the two groups are mutually dependent.

Delaporte (Trans. XVIth Int. Congr. Act., II, Brussels rg6o, pp. I27-I28) reports of a graduation (without test) in two groups of 
motor accidents based on a Polya process. Evidently, the agreement between observed and calculated number of vehicles with $n$ claims, $n=0, \mathrm{I} \ldots 5, \geq 6$ respectively $0, \mathrm{I} \ldots 9$ for the two groups respectively, is satisfactory for both groups, for one of the groups the agreement is excellent. (The deductions on page I23-124 of Delaporte's paper lead to Delaporte's formulae (I) and (2) for the moments of the unconditioned risk distribution. These formulae are, however, direct consequences of Lundberg's more general formulae, 1.c. (I22), (I22')).

7. To the judgement of the author of this note all investigations referred to do point to the conclusion that the empirical results in Sickness and Accident Statistics may be rationally approximated by Polya processes. The introduction of $(5 \mathrm{~b})$ as an alternative hypothesis has, so far, not improved the approximation. As the formulae for the Polya process are of a much simpler form, the author prefers the use of this process--in the sense of the principle of least square the "best" approximation to every compound Poisson processuntil it can be proved that other more complicated models afford a better fit.

One advantage of the Polya process not mentioned here above is that this process by the transformation $u=-\log P_{0}(t)=$ $t$

$\int_{0} p_{0}(v) d v$ can be transformed into a time homogeneous process. (Lundberg 1.c. pp. 57-58, 68). The transform is space heterogeneous (Philipson, Skand. Akt. Tidskr., I956, p. 36). The transform. for a non-elementary Polya process has a distribution function of the sum total of all changes having occurred in the parameter interval $(0, u)$, which may for every fix value of $u$ be asymptotically expanded in powers of $u^{\mathbf{1 / 2}}$ with a remainder term of the order of the first term neglected, on condition that for $u$ tending to infinity $b u$ remains finite, $b$ as before being the variance of $U(x)$, and that, in addition, the conditioned distribution of the size of a change relative to the hypothesis that one change has occurred contains only one absolutely continuous component and its moments exist up to an order needed for the expansion. (Ammeter, Skand. Akt. Tidskr., I948, p. I90; Philipson, Trans. XVth Int. Congr. Act., New York, 1957, p. 273). This statement holds also if the distribution function of the size of one change having occurred in the parameter 
point $u$, depends on $u$ (Philipson, 1.c. pp. 269-27I). Lundberg has (l.c. pp. 57-58, 97) proved that the Polya process is the only compound Poisson process with a homogeneous transform. Lundberg gives, further, (l.c. Pp. I2I-I22) an asymptotical expansion on certain conditions in the case where lim $b u$ is infinite for $u$ tending to infinity in powers of $t^{-1}$, in this case the expansion is based on Pearson density function Type III, while in the opposite case the expansions, described above, correspond to the expansions for the Poisson process, the normal approximation (Philipson) or the Esscher approximation (Ammeter), which have been described by Cramér (Skandia Jubilee Volume, r955, 4.2 and 4.3).

The two cases of the Polya process treated in the last paragraph correspond to the cases $\beta$ and $\alpha$ respectively as defined by Segerdahl in his survey in The Harald Cramér Volume (Uppsala I959, p. 297). Segerdahl gives for these cases, only the limits of the distribution function for accumulated gains, when, for case $\alpha, t$ and, for case $\beta, n$ tend to infinity. Only for his case $\gamma$ which refers to the Ammeter transform of several Polya processes mentioned in section I (c) of this note, Segerdahl has pointed out that the normal and the Esscher approximation to the distribution function may be used (l.c. p. 298). 